\title{
The effect of neutrophil/lymphocyte ratio on 1-year mortality in pulmonary embolism diagnosed patients.
}

\author{
Rıfat Urnal $^{1}$, Pınar Yeşim Akyol ${ }^{2 *}$, Zeynep Karakaya², Umut Payza², Fatih Esad Topal², Eylem Kuday \\ Kaykısız $^{3}$ \\ ${ }^{1}$ Department of Emergency Medicine, Palandöken State Hospital, Erzurum, Turkey \\ ${ }^{2}$ Department of Emergency Medicine, Izmir Katip Çelebi University Atatürk Training and Research Hospital, İzmir, \\ Turkey \\ ${ }^{3}$ Department of Emergency Medicine, Bitlis State Hospital, Bitlis, Turkey
}

\begin{abstract}
Background: Pulmonary embolism (PE) is one of the major causes of mortality, morbidity and hospitalizations. We aimed to investigate the effect of neutrophil/ymphocyte ratio (NLR) on 1-year mortality.

Material and methods: Among the patients, were referred to the emergency department of our hospital within 1-year who underwent computed tomography pulmonary angiography (CTPA), patients who were interpreted as PE after CTPA were included in the study.

Results: The optimal NLR cutoff value for the power in predict 1-year mortality was determined as 4.74 with ROC analysis. The NLR cutoff value of 4.74 had sensitivity of $61 \%$, specificity of $75 \%$. There were statistically significant differences between groups in terms of NLR on 1-year mortality and mortality in patients with a NLR greater than 4.74 was statistically significant higher than in patients with a NLR of 4.74 or less.
\end{abstract}

Conclusion: We believe that NLR can be used in evaluating 1-year mortality of pulmonary embolism.

Keywords: Pulmonary embolism, Neutrophil/lymphocyte ratio, Mortality.

Accepted on March 01, 2018

\section{Introduction}

Pulmonary Embolism (PE) is a common disease that can be fatal. According to the study conducted in the United States, more than 42 million deaths occurred in $20 \mathrm{y}$ and about 600 of these deaths $(1.5 \%)$ were diagnosed with PE [1]. PE is one of the major causes of mortality, morbidity and hospital admissions in all around the world. In 6 European countries, 317 thousand deaths were found associated with Venous Thromboembolism (VTE) occurred in 2004 (total population 454.4 million). In these cases, $34 \%$ of cases were presumed to be acute lethal PE, 59\% of deaths were not diagnosed with PE, only $7 \%$ were diagnosed with PE in the early period and early treatment could be given [2].

There are many environmental and genetic risk factors for PE. VTE arises from endogenous interactions of patient-related persistent risk factors and environmental transient risk factors. VTE may occur, throughout 6 weeks to 3 months prior to diagnosis and was induced by several risk factors such as surgery [3], trauma, immobilization, pregnancy [4], oral contraceptive use [5], cancer [6,7] or hormone replacement therapy [8] and VTE can also occur when there is no known risk factor. Smoking, obesity, high cholesterol, hypertension
(HT) and diabetes can be considered as direct or indirect common risk factors $[9,10]$.

Early and accurate diagnosis is vital in PE as well as in many emergency conditions. When the patients are diagnosed correctly and on time, mortality falls below $10 \%$. In general, the story, symptoms and clinical findings of PE diagnosis are highly sensitive but low specificity [11]. Clinical diagnosis is difficult. The most common symptoms are dyspnea, pleuritic pain, orthopnea, calf or thigh pain-swelling, wheezing resulted with rest or exertion [12]. Signs and symptoms of DVT are common [13].

$\mathrm{PE}$ is often asymptomatic that why both clinical monitoring and clinical prediction rules are useful in determining the likelihood of PE before the examination, although clinical evaluation alone is not reliable to detect or exclude PE diagnosis [14]. The only accepted rule for this process is the PE exclusion criteria (PERC rules) [13]. According to these criteria, patients with moderate or high risk are selected and further investigation is carried out in terms of PE.

The main goal of the diagnostic approach is to reduce the need for pulmonary angiography, is the gold standard for PE, as 
much as possible, which is invasive, expensive, not always easily accessible, and has certain morbidity and mortality for the treatment decision by using non-invasive methods as combination. For this purpose, different diagnostic algorithms including various diagnosis methods such as empirical clinical evaluation, d-dimer, lower extremity ultrasonography (USG), serial venous USG, ventilation-perfusion (V/Q) scintigraphy and computerized tomography pulmonary angiography (CTPA) $[15,16]$.

Containing pulmonary trunci or right and left pulmonary artery (PA) thrombosis is called central PE. PA branches may accompany the thrombus [17].

Various classifications have been developed to make it easier to decide whether patients to be hospitalized or discharge for the patients were diagnosed PE. These classifications will be useful both in determining which patient is at risk for premature death and therefore will require aggressive treatment and intensive care $[18,19]$, and also will determine which patients have a low risk of early complications such as death, venous thromboembolism, and major bleeding, and that we can discharge treat them [20]. The best-known indexes developed for this purpose are PE severity index (PESI) and Simplified PESI (SPESI).

Neutrophil/lymphocyte ratios (NLR) have been studied as a marker of acute inflammation in many areas. Studies of NLR values are available in the literature in acute abdomen cases such as mesenteric ischemia, peptic ulcer perforation, and acute cholecystitis, as lymph node metastasis marker in some cancers, in ischemic cases such as acute ischemic stroke, acute coronary syndromes, in vascular grafts such as aortic dissection, in rheumatologic diseases such as rheumatoid arthritis and ankylosing spondylitis, in COPD fever period and in various infectious conditions such as deep neck infection after acute tonsillitis [21-29].

The aim of this study was to form risk analysis by determining the risk factors that increase the mortality in this disease with high mortality and morbidity and to investigate the effect of NLR on mortality.

\section{Material and Methods}

Our retrospective cross-sectional study was initiated at the İzmir Kâtip Çelebi University Atatürk Training and Research Hospital after the approval of the ethics committee. Patients admitted to the Emergency Department within $1 \mathrm{y}$ and whose CTPA taken and patients diagnosed with PE after CTPA by two different radiologists were included in the study. Exclusion criteria were identified as patients with active infection before hospital admission, sepsis, leukemia, myeloproliferative disease, splenectomy with trauma history, pregnancy, below 18 $\mathrm{y}$ old and whose data could not be reached. A total of 66 patients meeting the inclusion criteria were screened retrospectively from the hospital system; demographic data such as age, sex, and additional illnesses, smoking status, symptoms of admission, vital signs at the time of admission, hemogram values and hospital admission or referral status were recorded. Neutrophil and lymphocyte counts were recorded by screening the hemograms that were taken from the patients at the time of admission and analyzed on the calibrated Mindray BC6800, which was sent to emergency biochemistry laboratory in vacuumed EDTA-contain K3 tubes. Neutrophil and lymphocyte counts accepted as normal within the range of 2-7 K/Ul and 0.8-4 K/Ul, respectively. NLRs were calculated and recorded. CTPA images were obtained with a Toshiba aquilion 64 multislice CT device after OmniPaque 300/100 mg contrast medium $1 \mathrm{mg} / \mathrm{kg}$ was given to the patients CTPA withdrawal. Images were evaluated by two blinded radiologists and patients with thrombus in the main PA or branches were considered as PE. Using CTPA, we defined central PE as presence of thrombus in the main PA or the left or right main PA. A peripheral PE was defined as thrombus only in the lobar, segmental, or sub-segmental PAs and not in the main PAs. The $1-y$ mortality due to pulmonary embolism was determined by records and communication with phone. The patients were divided into 2 groups according to their survival within $1 \mathrm{y}$. Patients who died within $1 \mathrm{y}$ were referred to as group 1, and patients who did not die within 1 y as group 2 . The relationship between recorded data and 1-y mortality was assessed.

\section{Statistical analysis}

The data obtained in this study were analyzed with the SPSS 20 packet program. Shapiro Wilk's has been exploited due to the unit numbers while investigating the normal distribution of variables. It is stated that the variables do not come from normal distribution when $\mathrm{P}<0.05$. Mann Whitney $\mathrm{U}$ Test was used because the variables were not different from the normal distributions while the differences between the groups were examined. Standardized z values for the Mann Whitney U Test are given since the number of units is more than 20. Chi-square analysis was applied when relations between groups of nominal variables were examined. Fisher's exact test was used when the expected values in $2 \times 2$ tables do not have sufficient volume. Pearson Chi-square analysis was employed to $\mathrm{R} \times \mathrm{C}$ tables with Monte Carlo Simulation. $\mathrm{P}<0.05$ was considered significant.

\section{Results}

A total of 94 patients admitted to emergency department within $1 \mathrm{y}$ and their CTPA was taken and diagnosed PE was identified. Exclusion criteria's were identified as patients with active infection before hospital admission, sepsis, leukaemia, myeloproliferative disease, splenectomy with trauma history, pregnancy, below 18 y old and whose data could not be reached. Twenty-eight patients excluded from the study because 8 of these had active infection or sepsis, 2 of these had myelodysplastic syndrome, 10 of these had not got a contact number in hospital system and the remaining 8 patient's information were incomplete in records. A total of 66 patients were included in the study and $35.84 \%(n=23)$ were male; $65.15 \%(n=43)$ were female of these patients. According to the sex, the mortality rate in males was $26.09 \%(n=6)$ and the mortality rate in females was $27.91 \%(n=12)$. There was no 
statistically significant difference in terms of sex status according to death status $(\mathrm{p}>0.05)$.

The mean age of the patients was 67.74. The average age of the patients who died within $1 \mathrm{y}$ was 75.22 , the average age of non-ex in 1 y was 64.94 and there was a statistically significant difference between the groups in terms of the patients' age according to their mortality status $(\mathrm{p}<0.05)$ (Table 1$)$. Mortality was higher in elderly patients.

Table 1. Mortality status of patients in terms of age.

\begin{tabular}{lllll}
\hline & $\mathbf{n}$ & Mean & Min-max & SD \\
\cline { 1 - 4 } Group 1 & 18 & 75.22 & $56-90$ & 9.65 \\
\cline { 1 - 4 } Group 2 & 48 & 64.94 & $24-92$ & 14.59 \\
\hline
\end{tabular}

Only 1 patient $(2.08 \%)$ was smoking; the remaining 65 patients $(97.92 \%)$ were not active smokers. It was determined that smoker patient did not die within a year.

When chronic illness cases of the patients included in the study were examined, $37(56.06 \%)$ patients had positive chronic

Table 2. Relationship between chronic disease status and mortality. illness status; 29 (43.94\%) patients had chronic disease status negative and $29.73 \%$ of the patients with chronic disease died within first $1 \mathrm{y} ; 72.73 \%$ of the patients did not die. When the effect of chronic disease was compared among the groups, no statistically significant difference was found between the groups with and without chronic disease in terms of mortality $(p>0.05)$. When chronic diseases of the patients were considered separately, $10(15.15 \%)$ patients had congestive heart failure (CHF), 13 (19.69\%) had coronary artery disease (CAD), 5 had chronic obstructive pulmonary disease (COPD) (7.57\%), 15 had hypertension (HT) $(22.72 \%), 5$ has diabetes mellitus (DM) (7.57\%) and $19(28.78 \%)$ had other diseases. According to these results, the most frequently diagnosed disease was HT, followed by CAD (19.69\%) and CHF $(15.15 \%)$. Chronic disease state and mortality relation are investigated; there was a statistically significant relationship between COPD status and mortality $(\mathrm{p}<0.05)$. $80 \%$ of the patients diagnosed with COPD were exitus and $77.05 \%$ of the non-COPD patients did not die (Table 2).

\begin{tabular}{|c|c|c|c|c|c|c|c|c|c|}
\hline & & \multicolumn{6}{|c|}{ Mortality } & \multicolumn{2}{|c|}{ Chi Square Test } \\
\hline & & \multicolumn{2}{|c|}{ Group 1} & \multicolumn{2}{|c|}{ Group 2} & \multicolumn{2}{|c|}{ Total } & \multirow[b]{2}{*}{ Chi Square } & \multirow[b]{2}{*}{$\mathbf{p}$} \\
\hline & & $\mathbf{n}$ & $\%$ & $\mathbf{n}$ & $\%$ & $\mathbf{N}$ & $\%$ & & \\
\hline \multirow{3}{*}{ Chronic disease } & No & 7 & 24.14 & 22 & 75.86 & 29 & 100 & \multirow{3}{*}{0.052} & \multirow{3}{*}{0.82} \\
\hline & Yes & 11 & 29.73 & 26 & 70.27 & 37 & 100 & & \\
\hline & Total & 18 & 27.27 & 48 & 72.73 & 66 & 100 & & \\
\hline \multirow{3}{*}{$\mathrm{CHF}$} & $\mathrm{CHF}$ negative & 14 & 25 & 42 & 75 & 56 & 100 & \multirow{3}{*}{ Fisher's exact } & \multirow{3}{*}{0.442} \\
\hline & CHF positive & 4 & 40 & 6 & 60 & 10 & 100 & & \\
\hline & Total & 18 & 27.27 & 48 & 72.73 & 66 & 100 & & \\
\hline \multirow{3}{*}{ CAD } & CAD negative & 14 & 26.42 & 39 & 73.58 & 53 & 100 & \multirow{3}{*}{ Fisher's exact } & \multirow{3}{*}{0.739} \\
\hline & CAD negative & 4 & 30.77 & 9 & 69.23 & 13 & 100 & & \\
\hline & Total & 18 & 27.27 & 48 & 72.73 & 66 & 100 & & \\
\hline \multirow{3}{*}{ COPD } & COPD negative & 14 & 22.95 & 47 & 77.05 & 61 & 100 & \multirow{3}{*}{ Fisher's exact } & \multirow{3}{*}{0.017} \\
\hline & COPD negative & 4 & 80 & 1 & 20 & 5 & 100 & & \\
\hline & Total & 18 & 27.27 & 48 & 72.73 & 66 & 100 & & \\
\hline \multirow{3}{*}{ HT } & HT negative & 13 & 25.49 & 38 & 74.51 & 51 & 100 & \multirow{3}{*}{ Fisher's exact } & \multirow{3}{*}{0.531} \\
\hline & HT positive & 5 & 33.33 & 10 & 66.67 & 15 & 100 & & \\
\hline & Total & 18 & 27.27 & 48 & 72.73 & 66 & 100 & & \\
\hline \multirow{3}{*}{ DM } & DM negative & 17 & 27.87 & 44 & 72.13 & 61 & 100 & \multirow{3}{*}{ Fisher's exact } & \multirow{3}{*}{1} \\
\hline & DM positive & 1 & 20 & 4 & 80 & 5 & 100 & & \\
\hline & Total & 18 & 27.27 & 48 & 72.73 & 66 & 100 & & \\
\hline Other & No & 11 & 23.4 & 36 & 76.6 & 47 & 100 & 0.647 & 0.421 \\
\hline
\end{tabular}




\begin{tabular}{lcccccc}
\hline Yes & 7 & 36.84 & 12 & 63.16 & 19 & 100 \\
\hline Total & 18 & 27.27 & 48 & 72.73 & 66 & 100 \\
\hline Dispatch & 16 & 27.12 & 43 & 72.88 & 59 & 100 \\
\hline Total & 18 & 27.27 & 48 & 72.73 & 66 & 100
\end{tabular}

When the arrival symptoms of the patients were examined, the most common symptom was dyspnea in $56 \%(n=37)$ followed by dizziness with $15.15 \%(n=10)$ and chest pain with $4.55 \%$ $(n=3)$. Other complaints included fever, leg pain, palpitation, blurring of consciousness, joint pain, general impairment, weakness, abdominal pain, seizures, syncope, back pain and pretibial edema. There was no statistically significant difference between the groups when the complaints of the patients and the mortality relation were examined $(\mathrm{p}>0.05)$.

In our study $66.7 \%(n=44)$ were peripheral and $33.3 \%(n=22)$ were central PA of the 66 patients, when the patients in the study group were compared in terms of being central or peripheral. The mortality rate of central embolism within $1 \mathrm{y}$ was $36.36 \% \quad(n=8)$ while the mortality rate of peripheral embolism was $22.73 \%(n=10)$ and there was a statistically significant relationship between being status of central or peripheral and death $(\mathrm{p}<0.05)$ (Table 3$)$.

Table 3. Comparison between groups according to central-peripheral state.

\begin{tabular}{|c|c|c|c|c|c|}
\hline & \multicolumn{2}{|c|}{ Group $1(n=18)$} & \multicolumn{2}{|c|}{ Group 2(n=48) } & \multirow[t]{2}{*}{$P=0.04$} \\
\hline & $\mathrm{n}$ & $\%$ & $\mathrm{n}$ & $\%$ & \\
\hline Central & 8 & 44.5 & 14 & 29.2 & \\
\hline Peripheral & 10 & 55.5 & 34 & 70.8 & \\
\hline Total & 18 & 100 & 48 & 100 & \\
\hline
\end{tabular}

Table 4. Comparison between groups in terms of association with NLR with a cut-off value of 4.7 and 1-y mortality.

\begin{tabular}{llll}
\hline & Group 2 $\mathbf{n}(\%)$ & Group 1 $\mathbf{n}(\%)$ & p value \\
\hline NLR $<4.74$ & $36(83.7)$ & $7(16.3)$ & 0.006 \\
\cline { 1 - 3 } NLR $>4.74$ & $12(52.2)$ & $11(47.8)$ & \\
\hline
\end{tabular}

7 of the patients diagnosed with PE were hospitalized and 2 died. It was determined that 16 of the 59 patients that were referred to the external center, died. NLR were examined when patients diagnosed with PE and the average of NLR was 5.46 $( \pm 5.05)$. When examined according to their mortality, NLR average of deaths was 7.52 and NLR averages of non-deaths was 4.68 within $1 \mathrm{y}$. When the NLR relationship with mortality of the patients was examined, NLR were found to be higher in the patients who died. But it was not statistically significant $(\mathrm{p}=0.06)$.

The optimal NLR cut-off value for the power in predict 1-y mortality was determined as 4.74 with ROC analysis and statistically significant (AUC: 0.65, \%95GA 0.47-0.84, LR
$+=2.2, \mathrm{p}<0.05)($ Figure 1$)$. The NLR cut-off value of 4.74 had sensitivity of $61 \%$, specificity of $75 \%$.

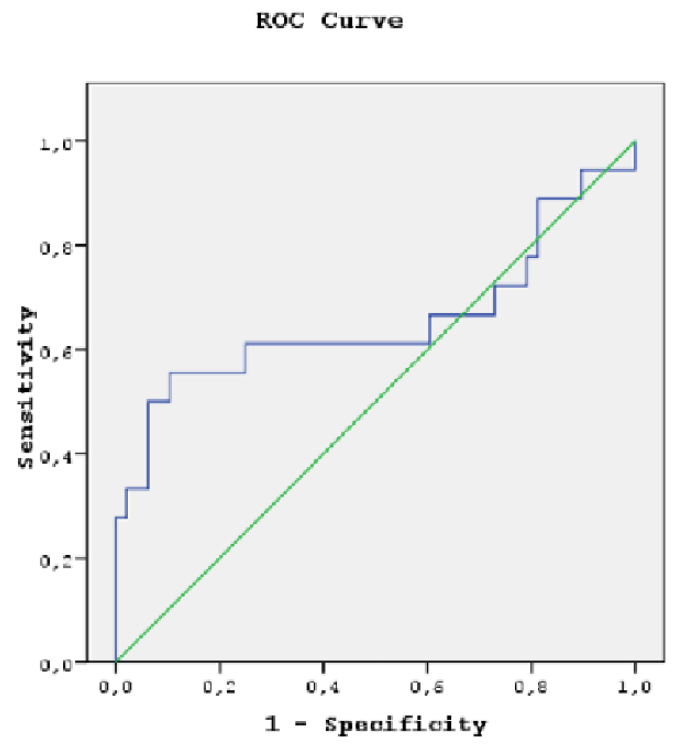

Figure 1. ROC analysis of NLR data for 1-y mortality. Optimal NLR cut-off value for 1-y mortality was determined as 4.74 (AUC=0.65).

$7(16.3 \%)$ of 43 patients with a NLR of 4.74 or less died, however $11(47.8 \%)$ of 33 patients had a NLR greater than 4.74 died. According to the chi-square statistical analysis, there was statistically significant differences between groups in terms of NLR on 1-y mortality and mortality in patients with a NLR greater than 4.74 was statistically significant higher than in patients with a NLR of 4.74 or less $(\mathrm{p}<0.05)$ (Table 4$)$.

\section{Discussion}

Despite the progress of diagnostic tests, it is difficult and timeconsuming to diagnose PE. Therefore, new researches are still being carried out and additional diagnostic tests are sought. The absence of a specific clinic and parameters of PE obliges physicians to synthesize clinical, laboratory and imaging to confirm the diagnosis of PE. In order for the clinician to quickly diagnose, it is necessary to choose an algorithm that is easily accessible and highly sensitive. A number of studies have been carried out and are currently being conducted in relation to the most appropriate diagnostic and therapeutic approaches. The need for risk identification and further investigation in patients with acute PE suspicion in emergency care is still a controversial issue and there is no consensus on the approach to the patients. Because of the limitations of laboratory methods, it is suggested that clinical considerations 
along with laboratory methods should be taken into consideration in cases with PE.

This study was performed among 66 patients who were diagnosed with PE. The average age of the participants was 67.74 and 23 of them were male $(34.85 \%)$ and $43(65.15 \%)$ were female. In the study conducted by Ergan et al. with 56 patients in 2016, the average age was 70.5 and the female and male ratio were $53.6 \%$ and $46.4 \%$, respectively [30]. Stein et al. found that PE frequency increased linearly with age and was more frequent in females over $50 \mathrm{y}$ of age [31]. On the other hand, conversely, Miniati et al. reported that male gender was one of the leading factors in increasing PE risk [16].

The incidence of venous thromboembolism increases with age and is valid for both idiopathic and secondary PE. PE is seen eight times higher in patients over $80 \mathrm{y}$ of age compared to those younger than $50 \mathrm{y}$ old. Mortality is usually associated with cancer, chronic cardiopulmonary comorbidity and high age [32]. In our study, when we assess the association of the 1$y$ mortality and age, the average age of the death patients within $1 \mathrm{y}$ is 75.2 and this age was found to be statistically significantly higher when compared to the age-average of nonex-patients. PE was more common in women; average age and age-mortality relation were determined in accordance with the literature. As the age increases, the mortality also increases proportionally.

Blondon et al. [33] in their study of the association between smoking and VTE published in 2015 have founded the conclusion that VTE is more common in smokers. Similar studies have been conducted on the effect of smoking on thrombosis [34,35]. However, the effect of smoking on the mortality of PE was not studied. Since there is only one patient in our study who smoked, it would be wrong to try to link the smoking with mortality and the 1-y mortality of the smoker patient is also negative.

In a study conducted by Tsai et al. [36], there was no statistically significant relationship between HT and PE when they investigated the relationship between chronic and PE risk. They found that there was a relationship between DM and PE in the same study. Conversely, there was no relationship between DM and PE in the study by Holst et al. [37]. Lee et al. [38] have investigated the risk variants associated with 1month mortality in PE and VT patients. However, there was no statistically significant correlation between HT, DM, CAD, and CHF and mortality with 1-month mortality. In the study of Ergan et al., the comorbidities most commonly associated with PE are HT and DM. They determined CHF, COPD, obesity and immobilization, trauma, and surgery as risk factors [30]. In this study, however, the relationship between mortality and chronic disease was not studied. In our study, the most common additional disease was HT and followed by CHF, CAD and COPD.

In elder age, PE can be masked in some diseases, such as acute coronary syndrome or chronic obstructive pulmonary disease (COPD) fever. The diagnosis of PE is difficult when it is associated with CHF or pneumonia [39]. The relationship between chronic disease and mortality was examined in our study; the reason that the mortality of COPD patients is statistically significantly high is due to the masking of pulmonary embolism and late diagnosis of COPD.

In a study conducted by Goldhaber et al., it was determined that $82 \%$ of PE cases had dyspnea, $49 \%$ had chest pain, $20 \%$ had cough, 14\% had fainting, and 7\% had hemoptysis [40]. In a study by Miniati et al. the most common distinctive clinical manifestations in patients with PE were dyspnea of $78 \%$, chest pain of $44 \%$ and fainting of $26 \%$ [16]. In our study, the most common symptom was dyspnea with $56 \%$, followed by chest pain, dizziness and fatigue, and our data were consistent with the literature.

In a study by Lee et al. [38], one-month mortality was not statistically significant in terms of mortality when embolism was center or pivotal. On the contrary, Jain et al. [41] in 2016 showed that 90-d mortality of patients with submassive embolism was statistically significantly higher than massive embolism. In our study, the 1-y mortality of patients with massive embolism was found to be statistically higher than that of patients with submassive embolism. In this respect, the data in the literature are contradictory and perspective multicentered studies are required.

In a study conducted by Soylu et al., it was found to be associated with an NLR of 10.8 times higher mortality rate in acute PE patients with a high NLR value more than 5.7 [42]. Kayrak et al. evaluated the relationship between increased NLR and prognosis in their studies in PE patients and that NLR at the time of admission to the hospital may be a 30-day mortality determinant in PE [43]. In our study, mortality rate was higher in patients with a NLR greater than 4.7 and this was compatible with the literature. This is also statistically significant. We are thinking that it will guide us in cases where PE is suspected, the NLR can be calculated to predict the mortality of this disease, which can progress rapidly and cause death, the diagnosis and treatment of the disease with hemogram measurement, which results in a short time after the emergency department visit and is easily accessible.

\section{Conclusion}

As the age increases, mortality due to pulmonary embolism increases. The most common comorbidity is HT. Patients with comorbid COPD, central embolism and an NLR of 4.74 have a higher mortality rate. We think that NLR can be used in evaluating 1-y mortality in order to determine the high-risk patients and to predict the mortality, which will be important in discharge and follow-up. Supportive studies in this subject, which will be carried out with prospective, multicenter, and more cases, are needed.

\section{Limitations}

The study has multiple limitations. First of all, owing to the retrospective nature of the study, comorbid diseases of patients could be assessed as much as they could be obtained from hospital data. Moreover, even if the data were written in full, 
additional illnesses reported by the patient and their relatives were included in the study. Another limitation is that the number of patients is very small and it is not right to reflect these results in a wider population. In addition to these, the main arrival symptom was not taken into consideration when arrival symptoms of the patients were evaluated. Finally, the effects of disease treatments on mortality have not been discussed. Due to all these limitations, studies with multiple center, prospective and wider patient population are needed.

\section{References}

1. Horlander KT, Mannino DM, Leeper KV. Pulmonary embolism mortality in the United States, 1979-1998: an analysis using multiple-cause mortality data. Arch Intern Med 2003; 163: 1711-1717.

2. Cohen AT, Agnelli G, Anderson FA, Arcelus JI, Bergqvist D, Brecht JG, Greer IA, Heit JA, Hutchinson JL, Kakkar AK, Mottier D, Oger E, Samama MM, Spannagl M; VTE Impact Assessment Group in Europe (VITAE). Venous thromboembolism (VTE) in Europe. The number of VTE events and associated morbidity and mortality. Thromb Haemost 2007; 98: 756-764.

3. Rogers MA, Levine DA, Blumberg N, Flanders SA, Chopra V, Langa KM. Triggers of hospitalization for venous thromboembolism. Circulation 2012; 125: 2092-2099.

4. Pomp ER, Lenselink AM, Rosendaal FR, Doggen CJ. Pregnancy, the postpartum period and prothrombotic defects: risk of venous thrombosis in the MEGAstudy. J Thromb Haemost 2008; 6: 632-637.

5. Blanco-Molina A, Rota LL, Di Micco P, Brenner B, Trujillo-Santos J, Ruiz-Gamietea A, Monreal M; RIETE Investigators. Venous thromboembolism during pregnancy, postpartum or during contraceptive use. Thromb Haemost 2010; 103: 306-311.

6. Timp JF, Braekkan SK, Versteeg HH, Cannegieter SC. Epidemiology of cancer associated venous thrombosis. Blood 2013; 122: 1712-1723.

7. Blom JW, Doggen CJ, Osanto S, Rosendaal FR. Malignancies, prothrombotic mutations, and the risk of venous thrombosis. JAMA 2005; 293: 715-722.

8. Sweetland S, Beral V, Balkwill A, Liu B, Benson VS, Canonico M, Green J, Reeves GK; Million Women Study Collaborators. Venous thromboembolism risk in relation to use of different types of postmenopausal hormone therapy in a large prospective study. J Thromb Haemost 2012; 10: 2277-2286.

9. Piazza G, Goldhaber SZ. Venous thromboembolism and atherothrombosis: an integrated approach. Circulation 2010; 121: 2146-2150.

10. Severinsen MT, Kristensen SR, Johnsen SP, Dethlefsen C, Tjønneland A, Overvad K. Smoking and venous thromboembolism: a Danish follow-up study. J Thromb Haemost 2009; 7: 1297-1303.

11. Okyay K, Cemri M, Cengel A. Acute pulmonary embolism. Anadolu Kardiyol Derg 2005; 5: 221-226.
12. Vanni S, Viviani G, Baioni M. Prognostic value of plasma lactate levels among patients with acute pulmonary embolism: the thrombo-embolism lactate outcome study. Ann Emerg Med 2013; 61: 330-338.

13. Albert RK. Slutsky A. Ranieri $M$ et al. Klinik Yogun Bakim 1. In: Goldman L. Ausiello D. (Cecil) Textbook of Medicine 22nd Ed. Baski (2010) Istanbul Tip Kitabevi Konu:3, Turkey.

14. van Belle A, Büller HR, Huisman MV, Huisman PM, Kaasjager K, Kamphuisen PW, Kramer MH, Kruip MJ, Kwakkel-van Erp JM, Leebeek FW, Nijkeuter M, Prins MH, Sohne M, Tick LW; Christopher Study Investigators. Effectiveness of managing suspected pulmonary embolism using an algorithm combining clinical probability, D-dimer testing, and computed tomography. JAMA 2006; 295: 172-179.

15. Miniati M, Prediletto R, Formichi B, Marini C, Di Ricco G, Tonelli L, Allescia G, Pistolesi M. Accuracy of clinical assessment in the diagnosis of pulmonary embolism. Am J Respir Crit Care Med 1999; 159: 864-871.

16. Turnier E, Hill JD, Kerth WJ, Gerbode F. Massive pulmonary embolism. Am J Surgery 1973; 125: 611.

17. Tapson VF. Acute pulmonary embolism. N Engl J Med 2008; 358: 1037-1052.

18. Kearon C, Kahn SR, Agnelli G, Goldhaber S, Raskob GE, Comerota AJ. Antithrombotic therapy for venous thromboembolic disease: American College of Chest Physicians Evidence-Based Clinical Practice Guidelines (8th Edition). Chest 2008; 133: 454S-545S.

19. Jime'nez D, Yusen RD, Otero R. Prognostic models for selecting patients with acute pulmonary embolism for initial outpatient therapy. Chest 2008; 132: 24-30.

20. Aktimur R, Cetinkunar S, Yildirim K, Aktimur SH, Ugurlucan M, Ozlem N. Neutrophil-to-lymphocyte ratio as a diagnostic biomarker for the diagnosis of acute mesenteric ischemia. Eur J Trauma Emerg Surg 2016; 42: 363-368.

21. Jung J, Park SY, Park SJ, Park J. Prognostic value of the neutrophil-to-lymphocyte ratio for overall and disease-free survival in patients with surgically treated esophageal squamous cell carcinoma. Tumour Biol 2016; 37: 7149-7154.

22. Dirican N, Karakaya YA, Günes S. Association of intratumoral tumor infiltrating lymphocytes and neutrophilto-lymphocyte ratio are an independent prognostic factor in non-small cell lung cancer. Clin Respir J 2017; 11: 789-796.

23. Zhou D, Wan Z, Fan Y. A combination of the neutrophil-tolymphocyte ratio and the GRACE risk score better predicts PCI outcomes in Chinese Han patients with acute coronary syndrome. Anatol J Cardiol 2015; 15: 995-1001.

24. Wang D, Zhang FH, Zhao YT. Association of polymorphism in ICAM-1 (K469E) and cytology parameters in patients' initial blood test with acute ischemic stroke. Genet Mol Res 2015; 14: 15520-15529. 
25. Kurtipek E, Bekci TT, Kesli R, Sami SS, Terzi Y. The role of neutrophil-lymphocyte ratio and platelet-lymphocyte ratio in exacerbation of chronic obstructive pulmonary disease. J Pak Med Assoc 2015; 65: 1283-1287.

26. Tekeoglu I, Gürol G, Harman H, Karakeçe E, Çiftçi İH. Overlooked hematological markers of disease activity in rheumatoid arthritis. Int J Rheum Dis 2016; 19: 1078-1082.

27. Onuk T, Güngör B, Karatas B. Increased neutrophil to lymphocyte ratio is associated with in-hospital mortality in patients with aortic dissection. Clin Lab 2015; 61: 1275-1282.

28. Bakshi SS. Letter to the Editor regarding "Predictive value of the neutrophil-to-lymphocyteratio in patients with deep neck space infection secondary to acute bacterial tonsillitis". Int J Pediatr Otorhinolaryngol 2015; 28: S0165-5876.

29. Ergan B, Ergün E, Çalışkan T, Aydın K, Tokur ME, Savran Y, Koca U, Cömert B, Gökmen N. Mortality Related Risk Factors in High-Risk Pulmonary Embolism in the ICU. Can Respir J 2016; 2016: 2432808.

30. Stein PD, Hsiu Ling H, Afzal A. Incidence of acute pulmonary embolism in a general hospital. Chest 1999; 116: 909-913.

31. Uresandi F, Blanquer J, Conget F, de Gregorio MA, Lobo JL, Otero R, Pérez Rodríguez E, Monreal M, Morales P. Guidelines for the diagnosis, treatment, and follow-up of pulmonary embolism. Arch Bronconeumol 2004; 40: 580-594.

32. Blondon M, Wiggins KL, McKnight B. The association of smoking with venous thrombosis in women: a populationbased, case-control study. Thromb Haemost 2013; 109: 891-896.

33. Wattanakit K, Lutsey PL, Bell EJ, Gornik H, Cushman M, Heckbert SR, Rosamond WD, Folsom AR. Association between cardiovascular disease risk factors and occurrence of venous thromboembolism. A time-dependent analysis. Thromb Haemost 2012; 108: 508-515.

34. Lutsey PL, Virnig BA, Durham SB, Steffen LM, Hirsch AT, Jacobs DR Jr, Folsom AR. Correlates and consequences of venous thromboembolism: The Iowa Women's Health Study. Am J Public Health 2010; 100: 1506-1513.
35. Tsai AW, Cushman M, Rosamond WD. Cardiovascular risk factors and venous thromboembolism incidence: the longitudinal investigation of thromboembolism etiology. Arch Intern Med 2002; 162: 1182-1189.

36. Holst AG, Jensen G, Prescott E. Risk factors for venous thromboembolism: results from the Copenhagen City Heart Study. Circulation 2010; 121: 1896-1903.

37. Lee JS, Moon T, Kim TH, Kim SY, Choi JY, Lee KB, Kwon YJ, Song SH, Kim SH, Kim HO, Hwang HK, Kim MJ, Lee YK. Deep Vein Thrombosis in Patients with Pulmonary Embolism: Prevalance, Clinical Significance and Outcome. Vasc Specialist Int 2016; 32: 166-174.

38. Goldhaber SZ. Pulmonary embolism. Lancet 2004; 363: 1295-1305.

39. Goldhaber SZ, Visani L, De Rosa M. Acute pulmonary embolism: clinical outcomes in the International Cooperative Pulmonary Embolism Registry (ICOPER) Lancet 1999; 353: 1386-1389.

40. Jain CC, Chang Y, Kabrhel C, Giri J, Channick R, Rodriguez-Lopez J, Rosovsky RP, Fogerty A, Rosenfield $\mathrm{K}$, Jaff MR, Weinberg I. Impact of pulmonary arterial clot location on pulmonary embolism treatment and outcomes (90 Days). Am J Cardiol 2017; 119: 802-807.

41. Soylu K, Gedikli O, Eksi A. Neutrophil-to-lymphocyte ratio for the assessment of hospital mortality in patients with acute pulmonary embolism. Arch Med Sci 2016; 12: 95-100.

42. Kayrak M, Erdogan HI, Solak Y. Prognostic value of neutrophil to lymphocyte ratio in patients with acute pulmonary embolism: a restrospective study. Heart Lung Circ 2013; 13: 1034-1032.

\section{*Correspondence to}

Pınar Yeşim Akyol

Department of Emergency Medicine

Izmir Katip Çelebi University Atatürk Training and Research Hospital

Turkey 